\title{
Rigorous Analysis on Ring-Doped-Core Fibers for Generating Cylindrical Vector Beams
}

\author{
Hyuntai Kim ${ }^{1}$, Youngchul Kwon ${ }^{1}$, Luis Alonso Vazquez-Zuniga ${ }^{1}$, Seung Jong Lee ${ }^{1}$, Wonil Park ${ }^{2}$, \\ Youngsu Ham², Suhyung Song ${ }^{2}$, Joong-Hwan Yang ${ }^{2}$, and Yoonchan Jeong ${ }^{1 *}$ \\ ${ }^{1}$ Laser Engineering and Applications Laboratory, Department of Electrical and Computer Engineering, \\ Seoul National University, Seoul 151-744, Korea \\ ${ }^{2} L G$ Display R\&D Center, 1007, Deogeun-Ri, Wollong-Myeon, Paju 413-811, Korea
}

(Received August 5, 2014 : revised October 27, 2014 : accepted October 27, 2014)

\begin{abstract}
We propose a novel active fiber design for selectively generating cylindrical vector beams (CVBs) or cylindrical vector modes (CVMs) which can be applied to conventional fiber lasers. A fiber is designed to have a ring-shaped core refractive index profile which can lead to the best overlap between the active dopant distribution profile and the lowest-order CVM (LCVM) field profile. Therefore, the overlap factor (OVF) of the LCVM becomes even higher than that of the fundamental mode. We emphasize that this condition cannot be satisfied by a conventional step-index core fiber (SICF) but by the ring-doped core fiber (RDCF). Because the lasing threshold is inversely proportional to the OVF, the LCVM can predominantly be stimulated even without going through special procedures to impose extra loss mechanisms to the fundamental mode. We numerically verify that the OVF of the LCVM with the doped ions can significantly exceed that of the fundamental mode if the proposed fiber design is applied. In addition, an RDCF of the proposed fiber design can also operate in a regime containing no higher-order modes besides the LCVM, so that it can selectively and efficiently generate the LCVM without being disrupted by the parasitic lasing of the higher-order modes. We highlight that an optimized RDCF can lead to a $>30 \%$ higher OVF ratio than a SICF having the same doped area. The proposed model is expected to be useful for enhancing the efficiency of generating CVBs in an all-fiber format.
\end{abstract}

Keywords : Cylindrical vector beam, Fiber laser, Ring-doped core fiber, Laser threshold, Overlap factor OCIS codes : (060.3510) Lasers, fiber; (160.5690) Rare-earth-doped materials; (230.5440) Polarizationselective devices

\section{INTRODUCTION}

Cylindrical vector beams (CVBs) have recently been receiving a considerable amount of research attention because of their unique and interesting capability of carrying orbital angular momentum [1]. In particular, CVBs can offer highly focused beam spots as well as dipole-like oscillating field patterns [2-6], which can open up opportunities for novel applications, such as optical trapping [2], microscopy [3, 4], plasmonic focusing [5], high-precision laser machining [6], etc.

To date there have been a variety of studies on generating CVBs mostly in free space, utilizing special optical components [7-11], such as prisms, crystals, polarizers, lenses, axicons, retarders, etc., or using a beam-combining technique [12]. Other studies are related with the CVB generation utilizing novel types of passive optical fibers, which include a coupling scheme to launch CVBs into a fiber by placing a polarizer directly inside the fiber [13], a formation of CVBs through a passive solid-core fiber having a modified core-index profile [14], a photonic crystal fiber with a gold nanowire [15], etc. Recently, it has been demonstrated that CVBs can also be generated directly from an active fiber, i.e. a fiber laser [16-18]. In this case, CVBs are formed via fiber cylindrical vector modes (CVMs), e.g., $\mathrm{TE}_{01}, \mathrm{TM}_{01}$, and $\mathrm{HE}_{21}$ modes, which are capable of maintaining their orbital angular momenta while propagating in the fiber. For example, a radially polarized beam was generated via an ytterbium $(\mathrm{Yb})$-doped fiber (YDF) laser assisted by a converging axicon [17]. Since optical fibers offer great flexibility and compactness over the bulk-optic counterparts, the generation of CVBs in an all-fiber format would be of great advantage. However, the fiber laser systems for generating CVBs that have been demonstrated so far

\footnotetext{
*Corresponding author: yoonchan@snu.ac.kr

Color versions of one or more of the figures in this paper are available online.
} 
[16-18] have limitations in terms of efficiency and practicality such that the laser efficiencies were significantly lower than that of the conventional YDF-based lasers, which can be over $80 \%$ [19], and the systems still encompassed a number of free-space optical components other than the fiber itself (e.g., free space coupling optics, long-pass filters, mirrors, polarizers, etc.) in order for the laser to operate in a CVM. It is worth noting that such limitations are due to the fact that they utilized gain fibers having a step-index core profile [16-18]. In fact, the use of a step-index core fiber (SICF) as a gain medium inevitably gives rise to a severe reduction of laser efficiency because the overlap factor (OVF) between the CVM and the doped region tends to be poor compared to the case with the fundamental mode $\left(\mathrm{HE}_{11}\right.$ mode) that is a non-CVM. In other words, the CVM of a SICF generally yields a ring-shaped field pattern, so that the CVM can only extract a limited fraction of energy mainly from the edge of the core. Consequently, the population inversion built up in the central part of the core does not contribute to the CVM lasing, thereby being wasted through amplified spontaneous emission (ASE) processes, which eventually gives rise to the significant reduction of the laser efficiency, as was observed in Refs. [16-18].

Here, we propose a novel ring-doped-core fiber (RDCF) design in order to facilitate the generation of a CVM in an all-fiber format $[20,21]$. Optimizing the overlap between the doped area and the desired fiber mode, we find that the overlap factor (OVF) of the lowest-order CVM (LCVM) with the doped ions can exceed that of the fundamental mode, thereby leading to lower lasing thresholds for the LCVM than that for the fundamental mode. In particular, the use of the RDCF significantly reduces the unwanted loss incurred by the ASE, due to the fact that it offers much enhanced mode overlap between the active ions and the LCVM field. Therefore, one can expect that it will lead to significant increase in the overall laser efficiency.

\section{THEORY}

In general, the lasing threshold $P_{\text {th }}$ of a fiber laser in terms of pump power is given by [21]

$$
P_{t h}=\frac{h \nu_{p} \eta}{2 \sigma_{s} \tau\left[1-\exp \left(-\alpha_{p} \gamma_{p} l\right)\right]} \frac{A_{d}}{\gamma_{s}},
$$

where $h v_{\mathrm{p}}$ is the pump photon energy, $\sigma_{\mathrm{s}}$ the emission cross-section of the doped ions at the signal wavelength, $\tau$ the active ion's upper-state fluorescence lifetime, $\eta$ the round-trip cavity loss, $l$ the length of the gain fiber, and $a_{\mathrm{p}}$ the absorption coefficient of the fiber at the pump wavelength, $A_{d}$ the effective doped area, and $\gamma_{s}$ and $\gamma_{p}$ the OVFs of the signal and pump waves with respect to the doped area, respectively. It is worth noting that $A_{d}$ is defined by

$$
A_{d}=\frac{\left(\iint N(x, y) d x d y\right)^{2}}{\iint N(x, y)^{2} d x d y},
$$

where $N(x, y)$ is an arbitrary function for the dopant density distribution in the transverse plane of the fiber. In addition, $\gamma_{\mathrm{s}}$ and $\gamma_{\mathrm{p}}$ are defined by

$$
\gamma_{s, p}=\frac{\iint N(x, y) \psi_{s, p} d x d y}{\bar{N} \iint \psi_{s, p} d x d y},
$$

where $\psi_{s, p}$ is the modal field intensity at the signal or pump wavelength, and $\bar{N}$ is the mean dopant density for the effective doped area $A_{d}$. If we combine Eqs. (2) and (3), assuming that the dopant density function is uniform over the doped region, the OVF can be simplified into

$$
\gamma_{s, p}=\frac{\iint_{\text {doped }} \psi_{s, p} d x d y}{\iint_{\text {total }} \psi_{s, p} d x d y} .
$$

As a result, the OVF becomes the ratio of the optical power confined in the doped region against the total optical power guided by the fiber.

For standard SICFs, the fundamental mode and the LCVM have clearly different field patterns: The former has a Gaussian-like field distribution whereas the latter has a ring-shaped one having a zero at the center as shown in Fig. 1, where the field intensity distributions of the fundamental

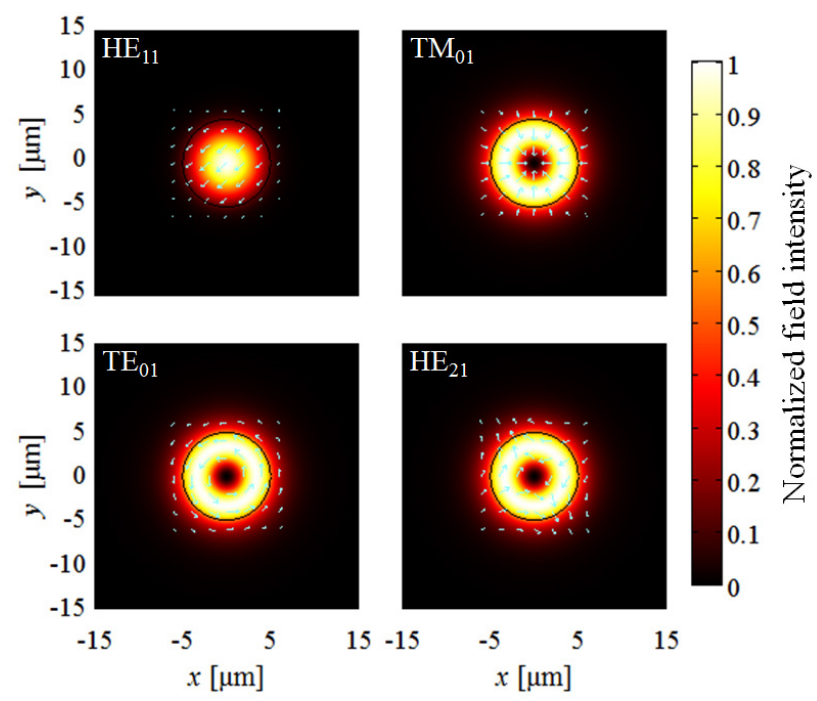

FIG. 1. The field intensity distributions of (a) the fundamental mode and (b)-(d) the LCVMs of a typical SICF when the core diameter is $9.9 \mu \mathrm{m}$. The arrowed lines denote the directions of the electric field vectors. 
mode and the LCVMs (TE $01, \mathrm{TM}_{01}$, and $\mathrm{HE}_{21}$ modes) of a typical SICF having a core diameter of $9.9 \mu \mathrm{m}$ and a core numerical aperture (NA) of 0.1 .

Thus, one can expect that if a fiber is given in an SICF form and its core is uniformly doped with active ions, the $\gamma_{\mathrm{s}}$ of the fundamental mode is invariably higher than that of the LCVM [22]. Consequently, the lasing threshold of the fundamental mode is always lower than that of the LCVM unless any additional mode discrimination mechanism is provided [21, 23] [see Eq. (1)]. In fact, this property can be a severe issue even with an additional mode discrimination measure because the excited ions in the central part of the core have poor overlap with the LCVM signal, so that their energy cannot be extracted efficiently, thereby causing an excessive level of the ASE build-up in the central part of the fiber. Actually, this consequence inevitably results in a significant reduction of the laser conversion efficiency [16-18].

To overcome such drawbacks of SICFs in generating a CVM, we propose to use an RDCF, introducing a dopantdepleted region at the center of its core. The central dopantdepleted region will prevent the unwanted ASE build-up, and also lead to a good mode overlap between the doped ions and the CVM field.

\section{NUMERICAL RESULTS}

\subsection{Limitations of a Step-index Core Fiber (SICF)}

Regarding the fibers under consideration, we utilize a finite-element-method (FEM)-based mode solver (COMSOL Multiphysics $^{B}$ ) to obtain the rigorous two-dimensional modefield distribution. We set the lasing wavelength of interest to be $1.06 \mu \mathrm{m}$, assuming that the fiber is doped with $\mathrm{Yb}$ ions. In addition, we fix the core NA to be 0.1 , which is a typical value for conventional YDFs having an intermediate mode size [24, 25].

Based on these fiber parameters, we first calculate the OVFs of the SICF modes, varying the core diameter. Then, we calculate the overlap factor ratio (OVFR) of the LCVM with respect to the OVF of the fundamental mode, i.e., $\gamma_{\mathrm{s}, \mathrm{LCVM}}$ $/ \gamma_{\mathrm{s}, \text { fund. }}$ In fact, this parameter can be an indicative that shows how efficiently the fiber is capable of generating the LCVM. For example, the higher the OVFR is, the higher efficiency the fiber will have in generating the LCVM. In particular, if the OVFR exceeds unity, the lasing threshold of the LCVM will be lower than that of the fundamental mode. In addition, it is worth noting that the LCVMs include $\mathrm{TE}_{01}, \mathrm{TM}_{01}$, and $\mathrm{HE}_{21}$ modes, depending of their polarization states, which are all degenerate modes and have nearly identical field intensity patterns under the weakly guiding condition, as shown in Fig. 1. Therefore in terms of the OVF there are no intrinsic differences among them, and one may represent their OVF properties with one representative value hereinafter.

Figure 2 shows the calculated results for the OVFs of

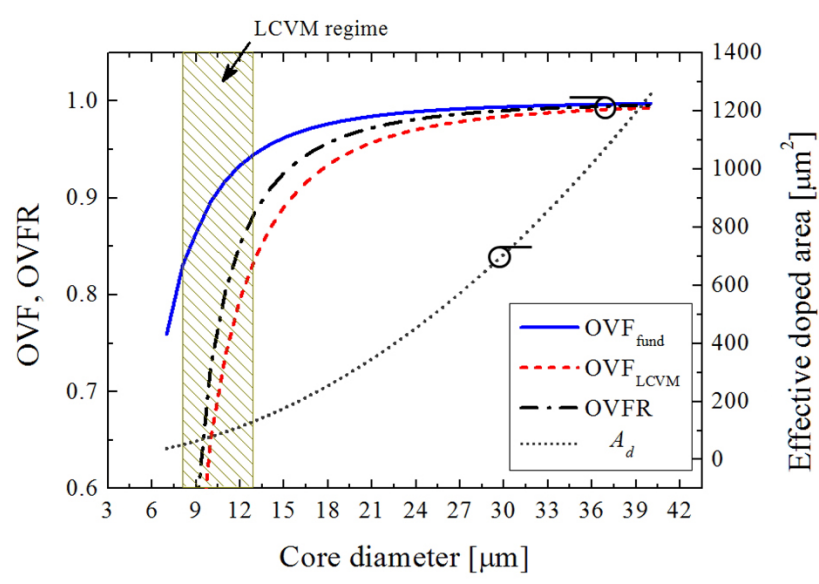

FIG. 2. The OVF for each mode, the corresponding OVFR, and the effective doped area $\left(A_{d}\right)$ of the SICF regarding to the diameter.

the fundamental mode and the LCVM, the corresponding OVFR between them, and the effective doped area $A_{d}$ as functions of the core diameter of the SICF. As expected, the results confirm that the OVF of the fundamental mode is invariably higher than that of the LCVM. Consequently, the fundamental mode is the fittest to survive during the mode completion in the laser cavity unless a sufficient special measure is provided to suppress it. In addition, when the core become larger, the OVF of the LCVM tends to converge to that of the fundamental mode. However, this does not mean any improvement for the LCVM generation in terms of the mode selection because as the core size increases, it also becomes a guide for other higher-order modes (HOMs), which in turn reduce the possibility of the LCVM generation [16, 26]. On this account, a desirable operating regime of a fiber is the case when it only supports modes up to the LCVM. The shaded region of Fig. 2 indicates the LCVM regime, when the core diameter is between 8.1 $\mu \mathrm{m}$ and $12.8 \mu \mathrm{m}$. The OVFR is maximized to 0.876 at the edge of this region when the core diameter is $12.8 \mu \mathrm{m}$. We can observe that the OVFR continuously increases after the LCVM regime. In other words, there is a tradeoff in increasing the OVFR or decreasing the number of modes in the case of a SICF.

\subsection{Design of a Ring-doped-core Fiber (RDCF)}

We now consider an active optical fiber having a core doped with laser-active ions, the refractive-index profile of which has an index well at the center, as depicted in Fig. 3, which is called an RDCF. We propose this structure because it can readily be implemented to a fiber via a modified chemical vapor deposition (MCVD) process [27, 28].

We assume the NA of the RDCF to be 0.1 as the same of the SICF investigated in the previous section. In fact, this NA will allow the RDCF to guide only the LCVM together with the fundamental mode if the effective mode area is scaled as similarly large as conventional YDFs 
having an intermediate mode size $[24,25]$.

We characterize the OVFs of the fundamental mode and the LCVM, varying $d_{\text {in }}$ and $\Delta r$, where $d_{\text {in }}$ denotes the inner diameter of the ring-doped core and $\Delta r$ its thickness in the radial direction, as depicted in Fig. 3. The refractive index profile (RIP) of the RDCF is also shown in Fig. 3. Fig. 4 shows typical field-intensity distributions of the modes guided in an RDCF for $d_{\text {in }}=18.5 \mu \mathrm{m}$ and $\Delta r=1.25 \mu \mathrm{m}$.

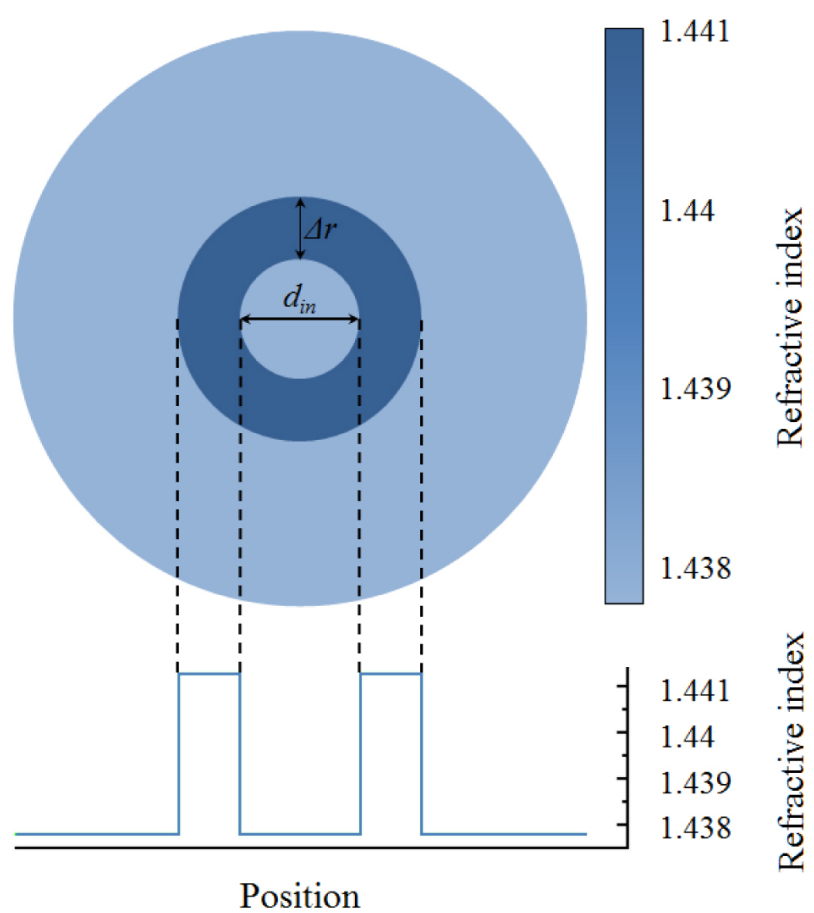

FIG. 3. The structure of the proposed RDCF.

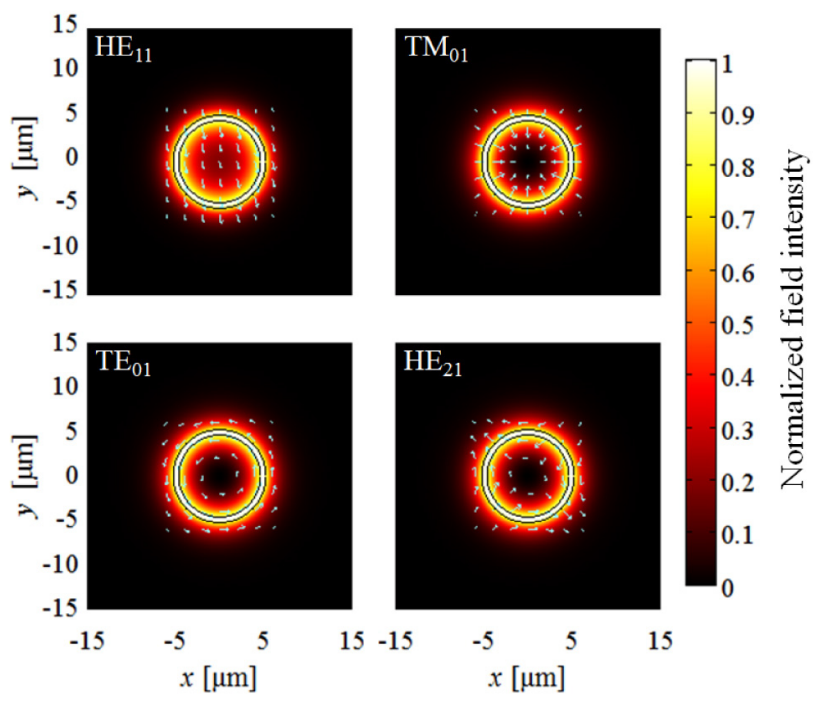

FIG. 4. The field intensity distributions of (a) the fundamental mode and (b)-(d) the LCVMs of the RDCF when $d_{i n}=18.5 \mu \mathrm{m}$ and $\Delta r=1.25 \mu \mathrm{m}$. The arrowed lines denote the directions of the electric field vectors.
It shows that in the case of the fundamental mode a considerable amount of the field still lies in the central part of the core, i.e., in the index well region where no laser-active ions are doped. In contrast, the field distribution of the LCVM is predominantly bounded within the ring-doped region, so that the field distribution in the index well region is significantly suppressed. Therefore, in this circumstance the OVF of the LCVM can be higher than that of the fundamental mode, and thus, the ASE build-up in the fundamental mode can significantly be reduced.

Figure 5(a) presents the OVFs of the fundamental mode (blue) and LCVM (red), and Fig. 5(b) presents the corresponding OVFR. In Fig. 5(a), one can see that a specific region exists where the red lines cover up the blue lines. This region represents the range where the OVF of the LCVM is higher than that of the fundamental mode. In particular, this range is highlighted by the color-graded lines in Fig. 5 (b), which actually represents the range where the OVFR exceeds unity. The shaded regions on the bottoms of Fig. $5(\mathrm{a})$ and 5(b) represent the parameter range allowing for operating in the LCVM regime. Figure 5(c) shows the calculated results for the OVFs of the fundamental mode and the LCVM, the corresponding OVFR, and the effective doped area $A_{d}$ in terms of $d_{\text {in, when }} \Delta r$ is fixed to $1.25 \mu \mathrm{m}$, for example. The shaded region of Fig. 5(c) also represents the parameter range for the LCVM regime. In contrast to the SICF case (see Fig. 2), one can see that OVFR can exceed unity even in the parameter range for the LCVM regime. This implies that within that specific parameter range the proposed fiber design can facilitate the LCVM operation rather than the fundamental-mode operation.

Figure 5(d) shows the field-intensity distributions of the fundamental mode and the LCVM across the diameter of an RDCF having its parameter set given by $d_{\text {in }}=18.5 \mu \mathrm{m}$ and $\Delta r=1.25 \mu \mathrm{m}$. This parameter set is indicated by a circle given in Fig. 5(a) or in Fig. 5(b). In fact, in this condition the OVFR is maximized within the range where the RDCF operates in the LCVM regime, supporting only the fundamental mode and the LCVM, which is eventually given by 1.026. In other words, it indicates the optimized design parameter set for the RDCF, which can lead to a lower lasing threshold for the LCVM than for the fundamental mode. Therefore, if the RDCF is pumped to lase, the LCVM can be the fittest to survive in the mode completion even without relying on any special mode-discrimination measure. Furthermore, in comparison with the case for the SICF, the RDCF yields considerably improved signal overlap with the doped ions even for the LCVM, so that the ASE loss due to the non-overlapped ions will be significantly reduced. This can eventually increase the overall laser efficiency. We emphasize this is the first practical fiber design to introduce higher gain to the LCVM than to the fundamental mode.

In addition, we would like to discuss a bit further the OVFR property of the optimized RDCF shown in Fig. 5(d), in comparison with various counterpart SICFs that are conventionally available. This comparison can be indicative 


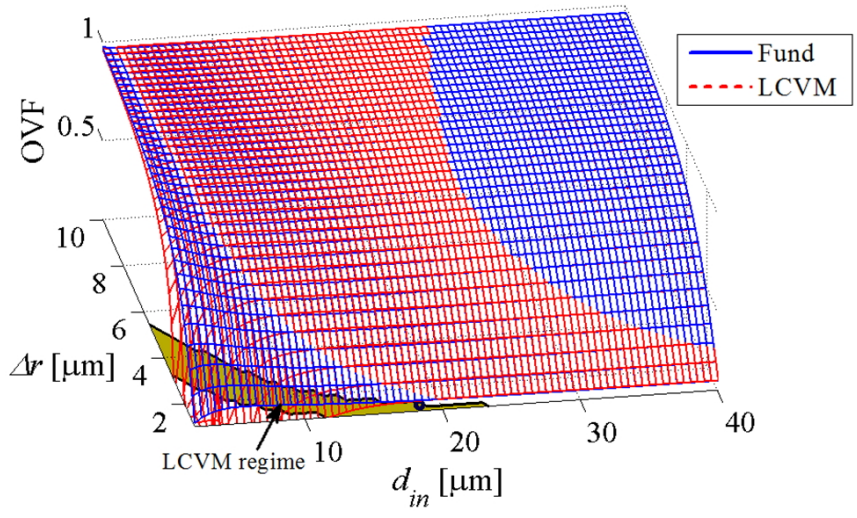

(a)

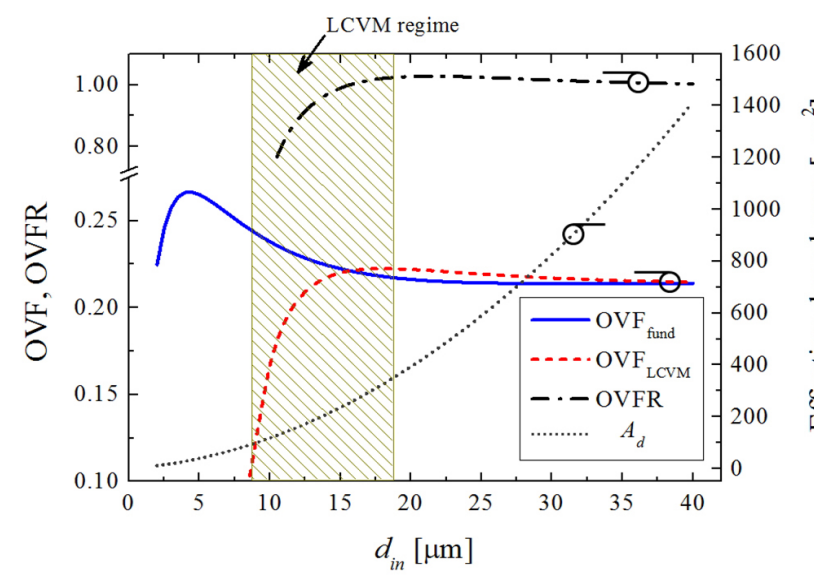

(c)

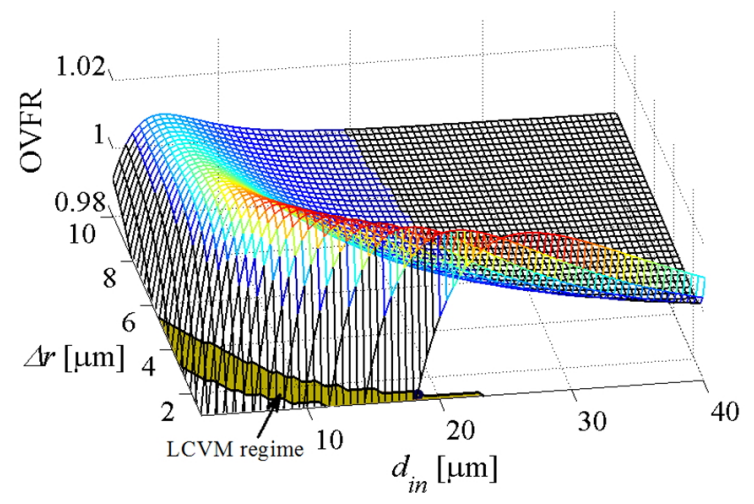

(b)

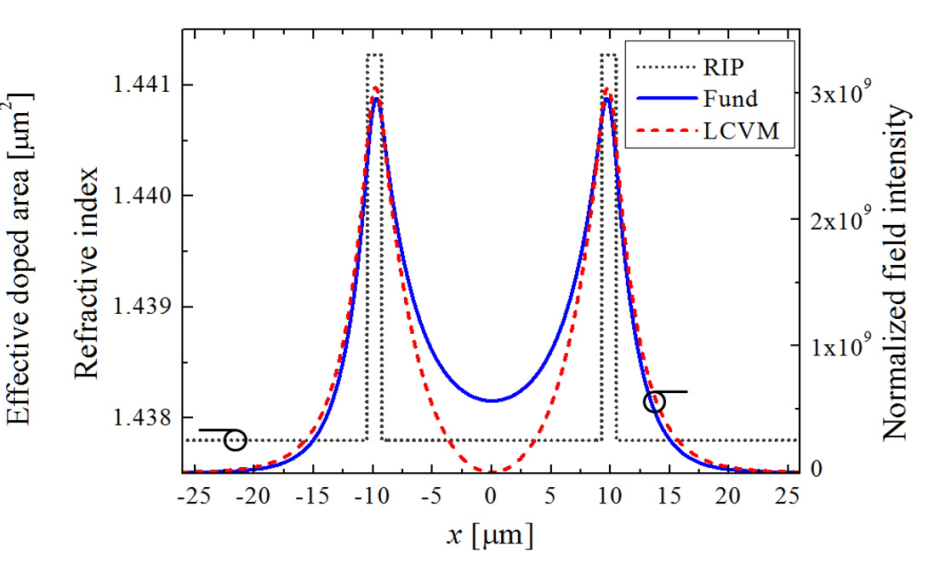

(d)

FIG. 5. (a) and (b): The OVF and the OVFR of the RDCF, respectively. (c) The OVF for each mode, the corresponding OVFR, and the effective doped area $\left(A_{d}\right)$ of the RDCF with respect to $d_{i n}$ when $\Delta r$ is fixed to $1.25 \mu \mathrm{m}$. (d) The field intensity distributions of the fundamental mode and the LCVM, together with the RIP of the RDCF when $d_{i n}=18.5 \mu \mathrm{m}$ and $\Delta r=1.25 \mu \mathrm{m}$.

TABLE 1. The characteristic parameters of the optimized RDCF and various SICFs

\begin{tabular}{l|c|c|c|c|c|c}
\hline \hline Fiber type & NA & $\begin{array}{c}\text { Core diameter } \\
{[\mu \mathrm{m}]}\end{array}$ & $\begin{array}{c}\Delta_{\text {OVFR }} \\
{[\%]}\end{array}$ & $\begin{array}{c}A_{\text {eff } L C V M} \\
{\left[\mu \mathrm{m}^{2}\right]}\end{array}$ & $\begin{array}{c}A_{d} \\
{\left[\mu \mathrm{m}^{2}\right]}\end{array}$ & $\begin{array}{c}\text { Operation regime } \\
(\text { number of modes })\end{array}$ \\
\hline RDCF & 0.1 & $\begin{array}{c}d_{i n}=18.5 \\
d_{\text {out }}=21.0\end{array}$ & +2.36 & 588.64 & 77.56 & $\mathrm{LCVM}(6)$ \\
\hline SICF1 & 0.1 & 9.9 & -28.96 & 152.07 & 77.56 & LCVM $(6)$ \\
\hline SICF2 & 0.1 & 30.5 & -0.97 & 588.64 & 728.70 & HOM (46) \\
\hline SICF3 $^{[16,17]}$ & 0.15 & 12.5 & -3.98 & 117.65 & 122.72 & HOM (20) \\
\hline SICF4 $^{[18]}$ & 0.06 & 30.0 & -4.42 & 690.80 & 706.86 & HOM $(16)$ \\
\hline
\end{tabular}

of how efficiently the RDCF is capable of generating the LCVM. For various fiber parameter sets, Table 1 summarizes their comparative characteristics, including the NA, the core diameter, the deviation of the OVFR from unity, i.e., $\Delta_{O V F R}$ that is given by $\Delta_{O V F R}=$ OVFR -1 , the effective mode area of the LCVM $A_{\text {eff,LCVM, }}$, the effective doped area $A_{d}$, and the operation regime of the fiber. SICF1 and SICF2 represent the cases having the same effective doped area $A_{d}$ and the same effective mode area $A_{\text {eff, } L C V M}$ with the RDCF, respectively. SICF3 and SICF4 are the active fibers based on the SICF design demonstrated in Refs. [16, 17] and [18], respectively. In particular, it is worth noting that SICF1 operates in the LCVM regime, which is the same modal operating condition as that of the optimized RDCF. In this respect, the $\triangle_{O V F R}$ value of SICF1 can be an imperative factor for making a fair comparison with the optimized RDCF. We highlight that the optimized RDCF offers a substantially higher $\Delta_{O V F R}$ value than that of SICF1, allowing for an improvement of over $30 \%$. Another advantage offered by the optimized RDCF is that its effective mode 
area for the LCVM, i.e., $A_{\text {eff }, L C V M}$, is substantially larger than that of SICF1, which is an extremely important factor for mitigating the undesirable nonlinear effects in the fiber, such as stimulated Raman scattering, stimulated Brillouin scattering, optical Kerr effect, etc. [24, 29-31]. While SICF2, SICF3, and SICF4 show better performance than SICF1 in terms of OVFR, one cannot expect the overall performance for generating the LCVM out of them will be in the same manner because they predominantly operate in HOM regimes. In other words, the mode competition among the LCVM and the HOMs will substantially exacerbate the overall laser performance and efficiency even if the LCVM lasing is possible by any chance.

\section{CONCLUSION}

We have proposed and designed a novel RDCF that can efficiently support the LCVM generation in an all-fiber format. Through rigorous numerical analyses of RDCFs, we found that unlike SICFs, RDCFs can make the OVF of the LCVM exceed that of the fundamental mode. For an optimized RDCF that has the effective mode area of the LCVM $\left(A_{\text {eff }, L C V M}\right)$ of $588.64 \mu^{2}$ and the effective doped area $\left(A_{d}\right)$ of $77.56 \mathrm{\mu m}^{2}$, its OVFR is eventually given by 1.023 , which can lead to single LCVM operation when it is pumped to lase. This OVFR value is over $30 \%$ higher than that of a SICF having the same doped area as the RDCF. We stress this is the first practical active fiber design that can introduce higher gain to the LCVM than to the fundamental mode. In fact, the proposed fiber design can prevent undesirable mode conversions to the fundamental mode or any higher-order modes, thereby being capable of suppressing the unwanted ASE loss owing to them. We expect this numerical study will be beneficial to the future research in designing or fabricating active fibers aimed for the generation of CVBs.

\section{ACKNOWLEDGMENT}

This work was supported in part by LG Display R\&D Center.

\section{REFERENCES}

1. Q. Zhan, "Cylindrical vector beams: From mathematical concepts to applications," Adv. Opt. Photon. 1, 1-57 (2009).

2. Q. Zhan, "Trapping metallic Rayleigh particles with radial polarization," Opt. Express 12, 3377-3382 (2004).

3. D. P. Biss and T. G. Brown, "Polarization-vortex-driven second-harmonic generation," Opt. Lett. 28, 923-925 (2003).

4. G. Bautista, M. J. Huttunen, J. Mäkitalo, J. M. Kontio, J. Simonen, and M. Kauranen, "Second-harmonic generation imaging of metal nano-objects with cylindrical vector beams,"
Nano Lett. 12, 3207-3212 (2012).

5. Q. Zhan, "Evanescent Bessel beam generation via surface plasmon resonance excitation by a radially polarized beam," Opt. Lett. 31, 1726-1728 (2006).

6. Q. Zhan and J. R. Leger, "Focus shaping using cylindrical vector beams," Opt. Express 10, 324-331 (2002).

7. Y. Kozawa and S. Sato, "Generation of a radially polarized laser beam by use of a conical Brewster prism," Opt. Lett. 30, 3063-3065 (2005).

8. P. B. Phua and W. J. Lai, "Simple coherent polarization manipulation scheme for generating high power radially polarized beam," Opt. Express 15, 14251-14256 (2007).

9. S. Quabis, R. Dorn, and G. Leuchs, "Generation of a radially polarized doughnut mode of high quality," Appl. Phys. B 81, 597-600 (2005).

10. A. V. Nesterov, V. G. Niziev, and V. P. Yakunin, "Generation of high-power radially polarized beam," J. Phys. D: Appl. Phys. 32, 2871-2875 (1999).

11. T. G. Euser, M. A. Schmidt, N. Y. Joly, C. Gabriel, C. Marquardt, L. Y. Zang, M. Förtsch, P. Banzer, A. Brenn, D. Elser, M. Scharrer, G. Leuchs, and P. St. J. Russell, "Birefringence and dispersion of cylindrically polarized modes in nanobore photonic crystal fiber," J. Opt. Soc. Am. B 28, 193-198 (2011).

12. S. C. Tidwell, D. H. Ford, and W. D. Kimura, "Generating radially polarized beams interferometrically," Appl. Opt. 29, 2234-2239 (1990).

13. E. Rawson, "Radial and tangential polarizers," U.S. Patent 3719415 (1973).

14. S. Ramachandran, P. Kristensen, and M. F. Yan, "Generation and propagation of radially polarized beams in optical fibers," Opt. Lett. 34, 2525-2527 (2009).

15. P. Uebel, M. A. Schmidt, M. Scharrer, and P. S. J. Russell, "An azimuthally polarizing photonic crystal fibre with a central gold nanowire," New J. Phys. 13, 063016 (2011).

16. J.-L. Li, K.-I. Ueda, A. Shirakawa, M. Musha, L.-X. Zhong, and Z.-M. Zhang, "39-mW annular excitation of ytterbium fiber laser with radial polarization," Laser Phys. Lett. 4, 814-818 (2007).

17. J.-L Li, K.-I. Ueda, M. Musha, A. Shirakawa, and Z.-M. Zhang, "Converging-axicon-based radially polarized ytterbium fiber laser and evidence on the mode profile inside the gain fiber," Opt. Lett. 32, 1360-1362 (2007).

18. S. Kanazawa, Y. Kozawa, and S. Sato, "High-power and highly efficient amplification of a radially polarized beam using an Yb-doped double-clad fiber," Opt. Lett. 39, 2857-2859 (2014).

19. Y. Jeong, A. J. Boyland, J. K. Sahu, S. Chung, J. Nilsson, and D. N. Payne, "Multi-kilowatt single-mode ytterbium-doped large-core fiber laser," J. Opt. Soc. Korea 13, 416-422 (2009).

20. E. Desurvire, Erbium-Doped Fiber Amplifiers Principles and Applications (Wiley \& Sons, INC., New York, USA, 1994).

21. M. J. F. Digonnet and C. J. Gaeta, "Theoretical analysis of optical fiber laser amplifiers and oscillators," Appl. Opt. 24, 333-342 (1985).

22. R. Selvas, J. K. Sahu, L. B. Fu, J. N. Jang, J. Nilsson, A. B. Grudinin, K. H. Ylä-Jarkko, S. A. Alam, P. W. Turner, and J. Moore, "High-power, low-noise, Yb-doped, claddingpumped, three-level fiber sources at $980 \mathrm{~nm}$," Opt. Lett. 28, 1093-1095 (2003). 
23. K. Okamoto, Fundamentals of Optical Waveguides (Academic Press, 2006), Chapter 3.

24. Y. Jeong, J. K. Sahu, D. N. Payne, and J. Nilsson, "Ytterbiumdoped large-core fiber laser with $1.36 \mathrm{~kW}$ continuous-wave output power," Opt. Express 12, 6088-6092 (2004).

25. J.-C. Rousseau and R. Sauvageon, "Method of controlling the etching and collapse of an MCVD tube by monitoring internal pressure," U.S. Patent 6131413 (2000).

26. S. Lee, L. A. Vazquez-Zuniga, D. Lee, H. Kim, J. K. Sahu, and Y. Jeong, "Comparative experimental analysis of thermal characteristics of ytterbium-doped phosphosilicate and aluminosilicate fibers," J. Opt. Soc. Korea 17, 182-187 (2013).

27. W. A. Gambling, D. N. Payne, and H. Matsumura, "Mode conversion coefficients in optical fibers," Appl. Opt. 14, 1538-1542 (1975).
28. J. E. Townsend, S. B. Poole, and D. N. Payne, "Solution doping technique for fabrication of rare-earth doped optical fibers," Electron. Lett. 23, 329-331 (1987).

29. P. Dupriez, A. Piper, A. Malinowski, J. K. Sahu, M. Ibsen, B. C. Thomsen, Y. Jeong, L. M. B. Hickey, M. N. Zervas, J. Nilsson, and D. J. Richardson, "High average power high repetition rate picosecond pulsed fiber master oscillator power amplifier source seeded by a gain-switched laser diode at $1060 \mathrm{~nm}$," IEEE Photon. Technol. Lett. 18, 1013-1014 (2006).

30. D. J. Richardson, J. Nilsson, and W. A. Clarkson, "High power fiber lasers: Current status and future perspectives," J. Opt. Soc. Am. B 27, B63-B92 (2010).

31. K. Park and Y. Jeong, "A quasi-mode interpretation of acoustic radiation modes for analyzing Brillouin gain spectra of acoustically antiguiding optical fibers," Opt. Express 22, 7932-7946 (2014). 